\title{
Chinese Medicine Jiedu Huayu Granules Reduce Liver Injury in Rats by Regulating T-Cell Immunity
}

\author{
Minggang Wang $\mathbb{D},{ }^{1}$ Dewen Mao $\mathbb{D}^{2},{ }^{2}$ and Hanmin Li $\mathbb{D}^{1,3}$ \\ ${ }^{1}$ Hubei University of Chinese Medicine, Wuhan, Hubei, China \\ ${ }^{2}$ The First Affiliated Hospital of Guangxi University of Chinese Medicine, Guangxi, China \\ ${ }^{3}$ Affiliated Hospital of Hubei University of Chinese Medicine, Wuhan, Hubei, China \\ Correspondence should be addressed to Hanmin Li; lihanmin69@126.com
}

Received 4 June 2019; Revised 1 September 2019; Accepted 31 October 2019; Published 27 November 2019

Academic Editor: Kenji Watanabe

Copyright (c) 2019 Minggang Wang et al. This is an open access article distributed under the Creative Commons Attribution License, which permits unrestricted use, distribution, and reproduction in any medium, provided the original work is properly cited.

\begin{abstract}
Liver injury, one of the causes of liver failure, is mainly due to T-cell-mediated immunity. Traditional Chinese medicine Jiedu Huayu granules are often used to suppress liver damage and improve liver function. The specific regulatory mechanism of Jiedu Huayu granules has not been fully studied, and its function in the immune system remains unclear. Therefore, in this study, the mechanism of Jiedu Huayu granules in the prevention of hepatic injury was studied in a rat model of hepatic injury induced by D-galactoside and lipopolysaccharide. The cytotoxic T lymphocytes (CTLs) in the peripheral blood were examined. Perforin, granule B, and PD1 expression in CTL increased after the induction of hepatic injury and could be reduced by Jiedu Huayu granules. Hepatic apoptotic factors OX62, FAS, and TNFR1 associated with CTL function were also reduced by Jiedu Huayu granules. These results suggested that Jiedu Huayu granules could inhibit the inflammatory response to relieve liver damage by mediating the T-cell immunity. Therefore, the discovery of the mechanism of action of Jiedu Huayu granules in the immune system could allow their use more effectively in clinical practice.
\end{abstract}

\section{Introduction}

The liver is an important organ with metabolic functions in the human body. In the process of combating foreign viruses and bacteria, the liver acts as the first line of defense through an inflammatory reaction to resist the antigen invasion. However, an excessive inflammatory reaction often leads to hepatocyte apoptosis, liver damage, liver disease, viruses, alcohol, and formation of lipid peroxidation products, and various drugs may cause hepatitis [1]. Severe hepatitis can even cause acute liver failure (ALF), and although liver transplantation can alleviate and improve the condition, ALF mortality can be as high as 50\% [2]. Viral hepatitis is an infectious disease caused by various hepatitis viruses. It can develop as acute hepatitis or chronic hepatitis. The patients may have clinical symptoms such as fever with jaundice, hepatomegaly, and liver damage. The patients with chronic hepatitis may develop liver fibrosis and end-stage cirrhosis with complications such as portal hypertension, liver failure, and increased incidence of liver cancer [3].

The D-galactoside and lipopolysaccharide-induced liver injury model induced in rats by toxic doses mimic viral hepatitis and is therefore widely used in drug screening studies against viral hepatitis and fulminant hepatic failure [4-6]. In this model, LPS stimulates Kupffer cells (specialized macrophages located in the liver) to secrete proinflammatory factors such as tumor necrosis factor TNF- $\alpha$ and various interleukins, and a series of inflammatory responses are the main causes of liver damage [7-9]. T-cell-mediated immune response is the main cause of liver injury. $\mathrm{CD}^{+}$ cytotoxic T lymphocytes (CTLs) can cause acute necrotizing liver disease, and its killing effect on cells is mainly achieved by releasing perforin and granule B [10]. In chronic viral hepatitis, a high expression of the immunosuppressive molecule PD-1 and an autoimmune function on cells can also be detected $[11,12]$. 
Jiedu Huayu granule is a traditional Chinese medicine consisting of 6 herbs, such as fungus Yin Chen, Hedyotis diffusa, red peony, rhubarb, turmeric, and scutellaria. A previous report demonstrated its successful use as a treatment against ALF as early as 20 years ago [13]. Previous studies showed that this drug can inhibit hepatocyte apoptosis by reducing intracellular protease expression, and it also has a palliative effect on hepatic encephalopathy complicated by liver injury [14]. Despite the evidence already reported on the effect of Jiedu Huayu granules, their specific regulatory mechanism on improving liver function has not been fully studied and remains still unclear [15]. Thus, our work focused on the evaluation of the effect of Jiedu Huayu granules on liver function, and our results suggested that it inhibited the inflammatory response to relieve liver damage by mediating the T-cell immunity.

\section{Materials and Methods}

2.1. Animals. Male rats ( $n=136,250 \pm 30$ grams) were purchased from the Hubei Experimental Animal Center (Hubei, China) and housed in a standard animal laboratory with a $12 \mathrm{~h}-12 \mathrm{~h}$ light-dark cycle. They were provided with water and standard rat chow ad libitum and randomly divided into three groups: control $(n=41)$, GalN/LPS-induced $\operatorname{ALF}(n=55)$, and treatment $(n=40)$.

\subsection{Establishment of ALF Model and Collection of Liver Tissue.} The ALF model was established as previously described [15], with slight modifications. Three days before GalN/LPS model establishment, rats in the treatment group were treated with Jiedu Huayu granules by gavage at a concentration of $8.8 \mathrm{~g} / \mathrm{kg}$ once every 12 hours for 6 days. The model group was treated with an equal volume of saline instead of traditional Chinese medicine according to the same protocol above. Approximately $24 \mathrm{~h}$ after GalN/LPS, rats were sacrificed and the liver was immediately perfused through the left ventricle with chilled saline containing $25 \mathrm{U} / \mathrm{mL}$ heparin. The liver tissue was harvested in tubes and immediately snap-frozen in liquid nitrogen. After $2 \mathrm{~h}$, the liver samples were transferred to $-80^{\circ} \mathrm{C}$ and stored at until analysis. Serum biochemistry and liver histopathology were used to assess liver injury.

2.3. Chinese Herbal Formula. Jiedu Huayu granules contain Yin Chen 30 g, Hedyotis diffusa 30 g, Radix Paeoniae rubra $50 \mathrm{~g}$, rhubarb $15 \mathrm{~g}$, Tulip $15 \mathrm{~g}$, and Acorus tatarinowii $15 \mathrm{~g}$. Traditional Chinese herb granules manufactured by Jiangyin Tian Jiang Pharmaceutical Co. Ltd. (Jiangsu, China) were purchased from the First Affiliated Hospital of Guangxi University of traditional Chinese Medicine. Quality control of Jiedu Huayu granule prescriptions was performed in accordance with a published article [15].

2.4. Flow Cytometry. Cells were washed in PBS twice and stained for cell surface CD3 or CD8 on ice for 20 min in PBS plus anti-CD3-FITC and anti-CD8-APC (BD). Stained cells were sorted using a BD FACSAria and analyzed using a BD LSRFortessa. FlowJo software (FlowJo LLC, Ashland, OR) was used to perform the flow cytometry analysis.

2.5. Western Blotting. Western blot analysis was performed as described. Cells were lysed on ice for $30 \mathrm{~min}$. Approximately $50-150 \mathrm{mg}$ of thermally denatured protein extract was loaded on a $10 \%$ polyacrylamide gel, electroblotted onto a nitrocellulose membrane, and blocked for one hour. The membrane was then incubated with antibodies against perforin, granule B, and PD1. Bands were visualized using the ECL Western blotting system (Santa Cruz Biotechnology).

2.6. Immunohistochemistry. Selected liver sections were deparaffinized, rehydrated, and heated in a microwave oven in 0.01 M citrate buffer ( $\mathrm{pH}$ 6.0; Química Contemporânea, Diadema, Brazil) for $30 \mathrm{~min}$. Endogenous peroxidase activity was blocked by $3 \%$ hydrogen peroxide for $10 \mathrm{~min}$, followed by a wash with PBS. Sections were incubated overnight at $4^{\circ} \mathrm{C}$ with the following primary antibodies: anti-FAS $(1: 50)$, anti-OX62 (1:10), and anti-TNFR1 (1:100). The primary antibody was then detected using avidin-biotin peroxidase detection solution (DakoCytomation labelled streptavidin biotin reagent; DakoCytomation, Glostrop, Denmark; and System-horseradishperoxidase; Dako, Glostrop, Denmark), and the signal was visualized using diaminobenzidine (DakoCytomation) and Substrate Chromogen System (Dako). Slides were counterstained with Harris's hematoxylin, dehydrated, cleared, and mounted. Positive controls from the appendix and tonsils were used. Cells were initially observed at a low magnification $(\times 100)$ to assess the general distribution of the primary antibody. Samples were subsequently examined at a higher magnification $(\times 400)$ [16]. Liver cells (exhibiting gross and evident nucleoli and irregular chromatin) were identified, and stained cells were counted at the higher magnification.

2.7. Real-Time Quantitative PCR (RT-qPCR). cDNA templates were prepared after RNA extraction and reverse transcription. Amplification was performed on a real-time PCR system (Applied Biosystems 7500, USA). The whole procedure was according to the manual of SYBR ${ }^{\circledR}$ Premix Ex $\mathrm{Taq}^{\mathrm{TM}}$ kit (Takara RR420A, Japan). Relative expression was calculated using formula $1 / 2 \triangle \triangle$ Ct. Primers used for detecting the different expression of specific genes were as follows: GAPDH: forward 5'-ACAGCAACAGGGTG-

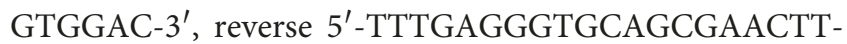
$3^{\prime}$. perforin: forward $5^{\prime}$-GCTGGATGTGAACCCTAAACC3, reverse $5^{\prime}$-GGAGCTGTTAAAGTTGTGGGG -3'. GrB: forward $5^{\prime}$-AGTGTGGCGGCTTCCTTATA-3', reverse $5^{\prime}$ TATACGCTGGGTGGGGAATG-3'. PD1: forward $5^{\prime}$-GGTATGTCAGAGGCCAGAGAA-3', reverse $5^{\prime}$-AATGGTGGCGTATTCTGTGTG - $3^{\prime}$.

2.8. Statistical Methods. Data were subjected to statistical analysis using the statistical package for social sciences 


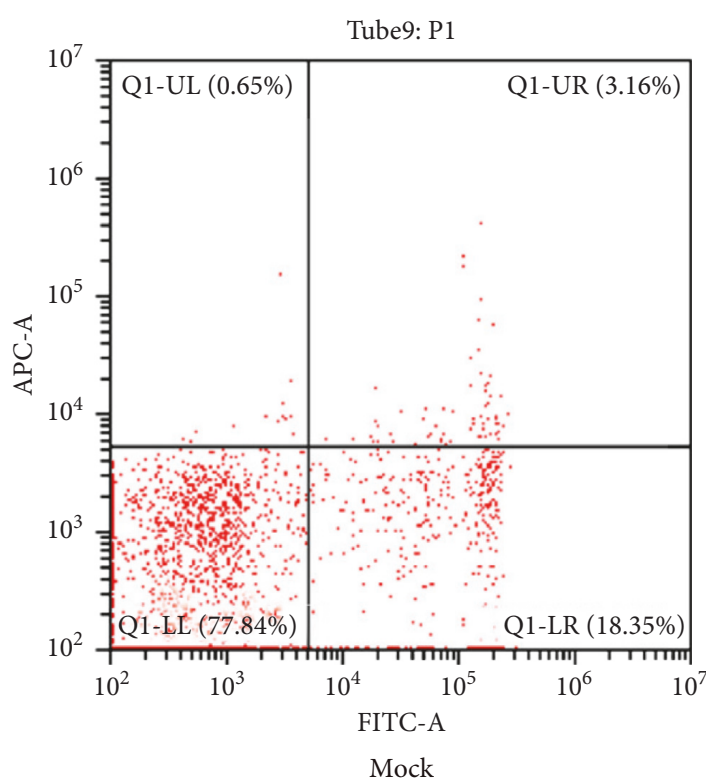

(a)

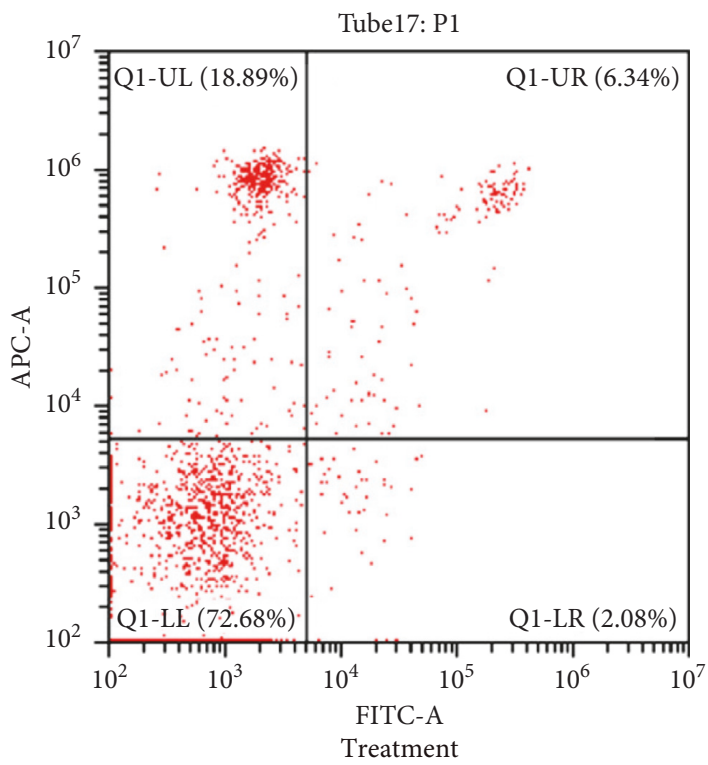

(c)

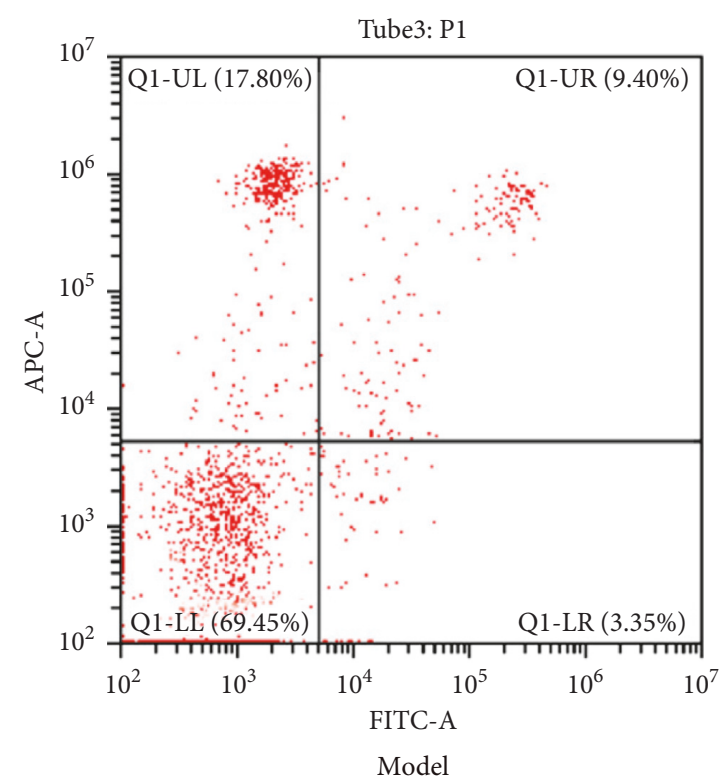

(b)

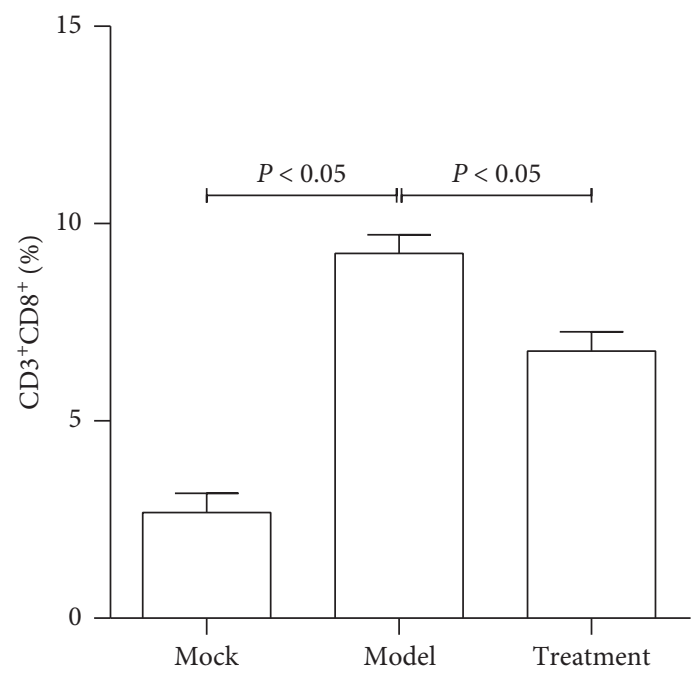

$\mathrm{CD}^{+}{ }^{+} \mathrm{CD} 8^{+}$

(d)

Figure 1: Effect of Jiedu Huayu granules on rat CTL cells. (a-c) $\mathrm{CD}^{+} \mathrm{CD} 8^{+}$cells from control group, model group, and treatment group by flow cytometry. The proportion of $\mathrm{CD} 3^{+} \mathrm{CD} 8^{+}$cells was detected at the same time. (d) Bar graph showing $\mathrm{CD} 3^{+} \mathrm{CD} 8^{+}$cell percentage in the three groups.

(SPSS), version 18.00. A one-way ANOVA or Student's $t$-test was used to determine statistical significance. A probability of $P<0.05$ was considered statistically significant. All data are presented as mean $\pm \mathrm{SD}$.

\section{Results}

3.1. Jiedu Huayu Granules Chinese Medicine Reduced the Proportion of CTL Cells. In patients with ALF, CTL mediated by immune function can lead to an overimmune response, leading to liver failure. Therefore, in order to detect the changes in CTL cells in ALF and Jiedu Huayu granule treatment, six rats were randomly selected from each group to detect the proportion of CTL cells in peripheral blood cells by flow cytometry. The results showed that, in the control group, the percentage of $\mathrm{CD}^{+} \mathrm{CD} 8^{+} \mathrm{CTL}$ cells in rat peripheral blood was $3.16 \%$, while in the ALF model group, the percentage of $\mathrm{CD} 3^{+} \mathrm{CD} 8{ }^{+}$CTL cells increased to $9.40 \%$. After treatment with Jiedu Huayu granules, the percentage of $\mathrm{CD}^{+} \mathrm{CD}^{+} \mathrm{CTL}$ cells decreased significantly to $6.34 \%$ (Figure 1). These experimental results have been described in our previous article [17] and indicate that Jiedu Huayu granules can reduce the proportion of CTL cells in the process of alleviating ALF, suggesting that Jiedu Huayu 

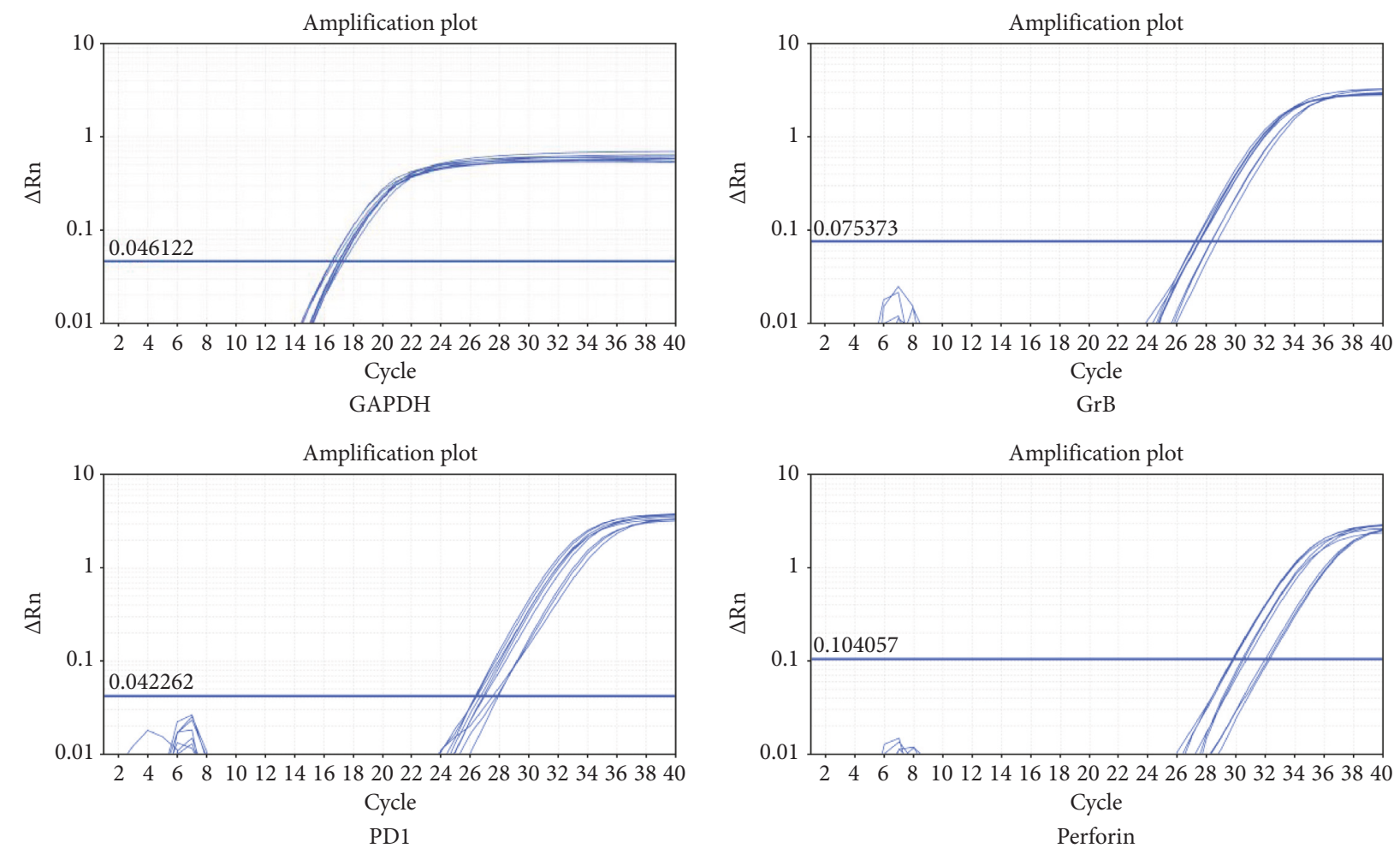

Amplification plot

(a)

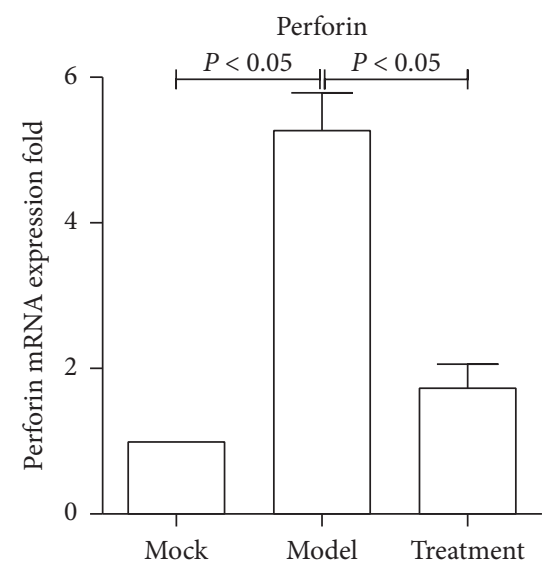

(b)

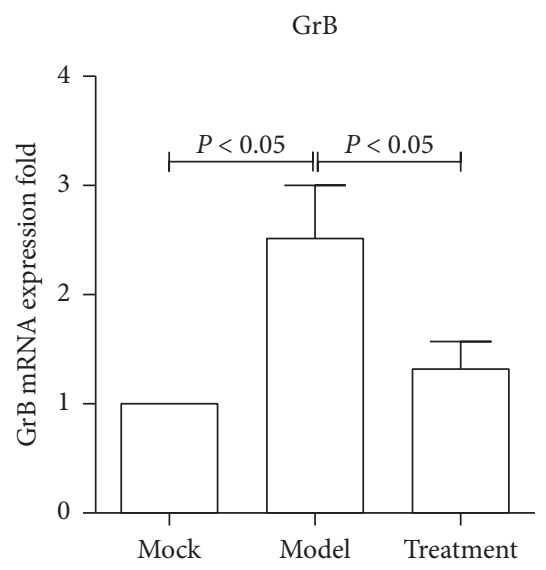

(c)

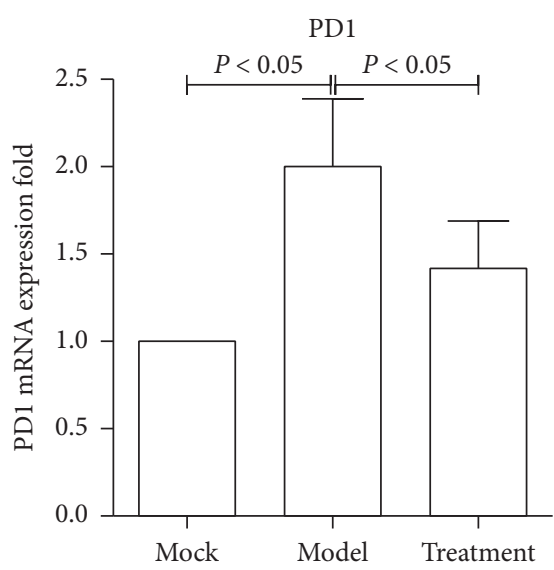

(d)

FIGURE 2: The expression of genes related to cell killing was detected by RT-qPCR. CD $3^{+} \mathrm{CD} 8^{+}$cells were isolated, and total RNA was extracted. The expression of perforin, GrB, and PD1 mRNA was detected by RT-qPCR after reverse transcription. Each experiment was repeated three times.

granules can alleviate excessive immune stress by reducing the ratio of CTL cells in the pathological process of acute liver injury.

\subsection{Jiedu Huayu Granule Chinese Medicine Inhibited CTL} Function. Ten rats from the control group, model group, and treatment group were killed, and the peripheral blood was collected. CTLs were sorted by $\mathrm{CD}^{+} \mathrm{CD}^{+}$cell labelling in the peripheral blood. The selected CTLs were analyzed, revealing that perforin, granule $\mathrm{B}$, and $\mathrm{PD}-1 \mathrm{mRNA}$ and protein expression significantly increased. Perforin, granule
B, and PD-1 expression was downregulated in the treatment group, indicating that the Chinese medicine improved ALF symptoms by inhibiting CTL function (Figures 2 and 3). In addition, these results suggested that Jiedu Huayu granules affected the killing efficacy of CTLs, thus alleviating CTLmediated immune damage.

3.3. Inhibition of Hepatocyte Apoptosis and Liver Immune Response. Next, to detect apoptosis and immune response in liver tissue, three rats from the control group, model group, and experimental group were randomly selected and 


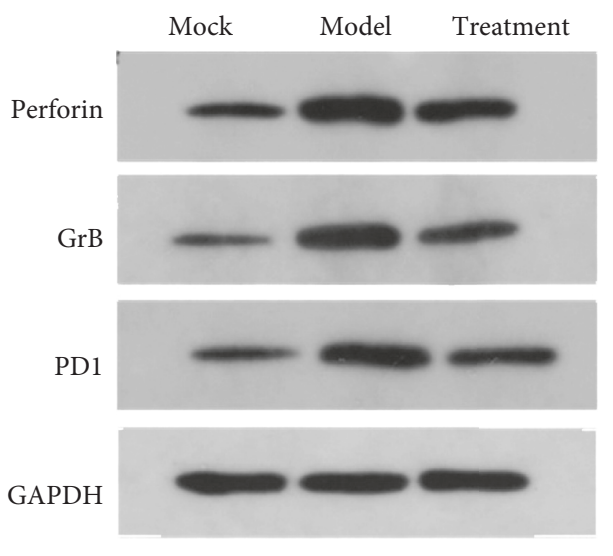

(a)

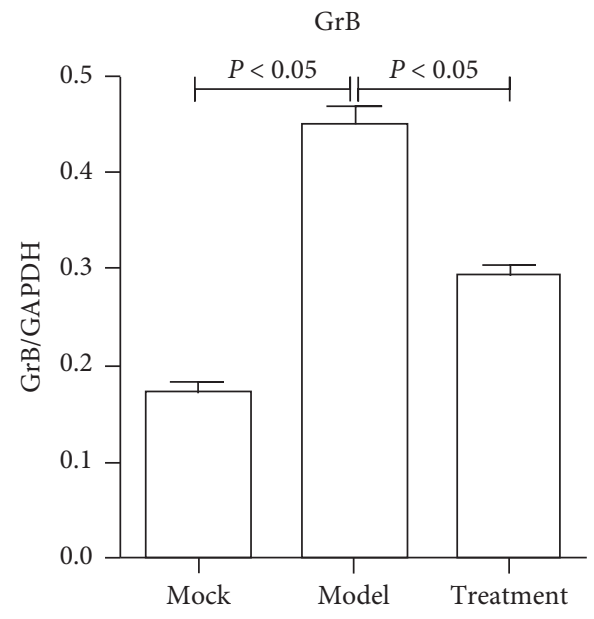

(c)

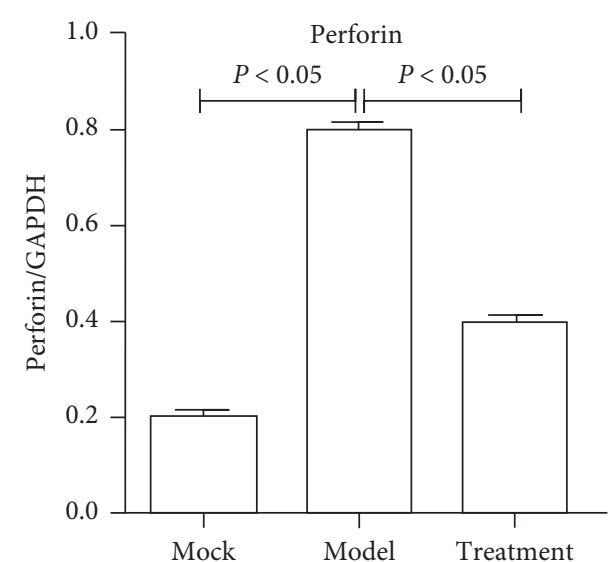

(b)

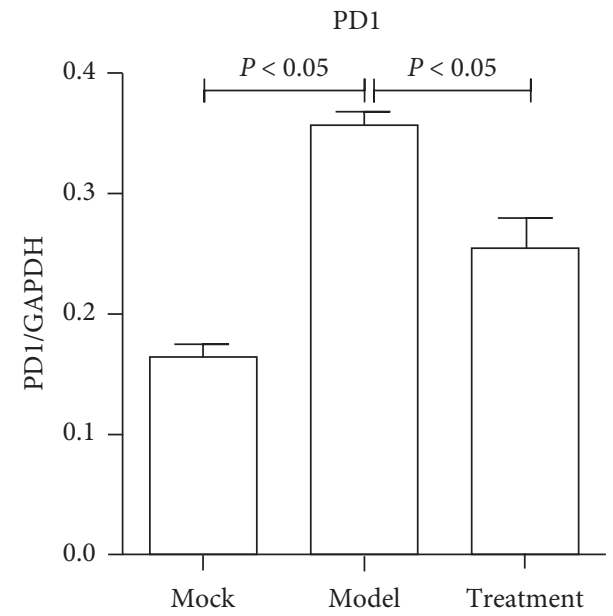

(d)

Figure 3: The expression of proteins related to cell killing was detected by using western blot. $\mathrm{CD} 3^{+} \mathrm{CD} 8^{+}$cells were isolated and total proteins were extracted. Perforin, GrB, and PD1 protein expression was detected by using western blot. Quantification of the gray bands was performed using BandScan software.

sacrificed. The liver tissue was collected and sliced, and immunohistochemical analysis to detect hepatocyte apoptosis related proteins OX62, FAS, and TNFR1 was performed. The results showed that these proteins were highly expressed in the model group. The signal intensity in the treatment group was similar to that in the control group, suggesting that the treatment with Jiedu Huayu granules reduced liver cell apoptosis (Figure 4). These experimental results are also described in our previous article [18]. It is well known that CTL cells can induce apoptosis through FAS and TNFR1, while OX62 is closely related to maturity of CTL cells. Thus, these results suggested that Jiedu Huayu granules reduced the expression of OX62, therefore affecting the maturation of CTL. In addition, it reduced the expression of FAS and TNFR1 to inhibit hepatocyte death, which is associated with the mechanism of Jiedu Huayu granules inhibiting CTL function.

\section{Discussion}

Immune damage can be triggered by multiple factors and is the main cause of liver failure. The immune system protects against the invasion of various pathogens and maintains the stability of the internal environment. However, under pathological conditions, excessive activation or incorrect activation of the immune system can be deleterious to the body [19]. The liver is not only the biochemical plant of the body but also an endocrine organ. Some scientists also regard the liver as an immune organ; thus, the liver is often the first to bear the brunt when the immune system damages the body. The chemical damage and immune damage of hepatocytes often occur at the same time. They complement each other under physiological conditions, protecting the body against the invasion of pathogenic microorganisms and other damages. However, under pathological conditions, they cause a vicious circle and aggravate liver cell damage [20].

In this work, the role of Jiedu Huayu granules in the immune system against liver injury was explored. As a traditional Chinese medicine, it is interpreted as detoxification and used in severe cases of heat and jaundice, severe hepatitis, hyperbilirubinemia, and other diseases [21]. However, the interpretation of Chinese medicine regarding the effect of Jiedu Huayu granules is not giving any information regarding the mechanism of action 

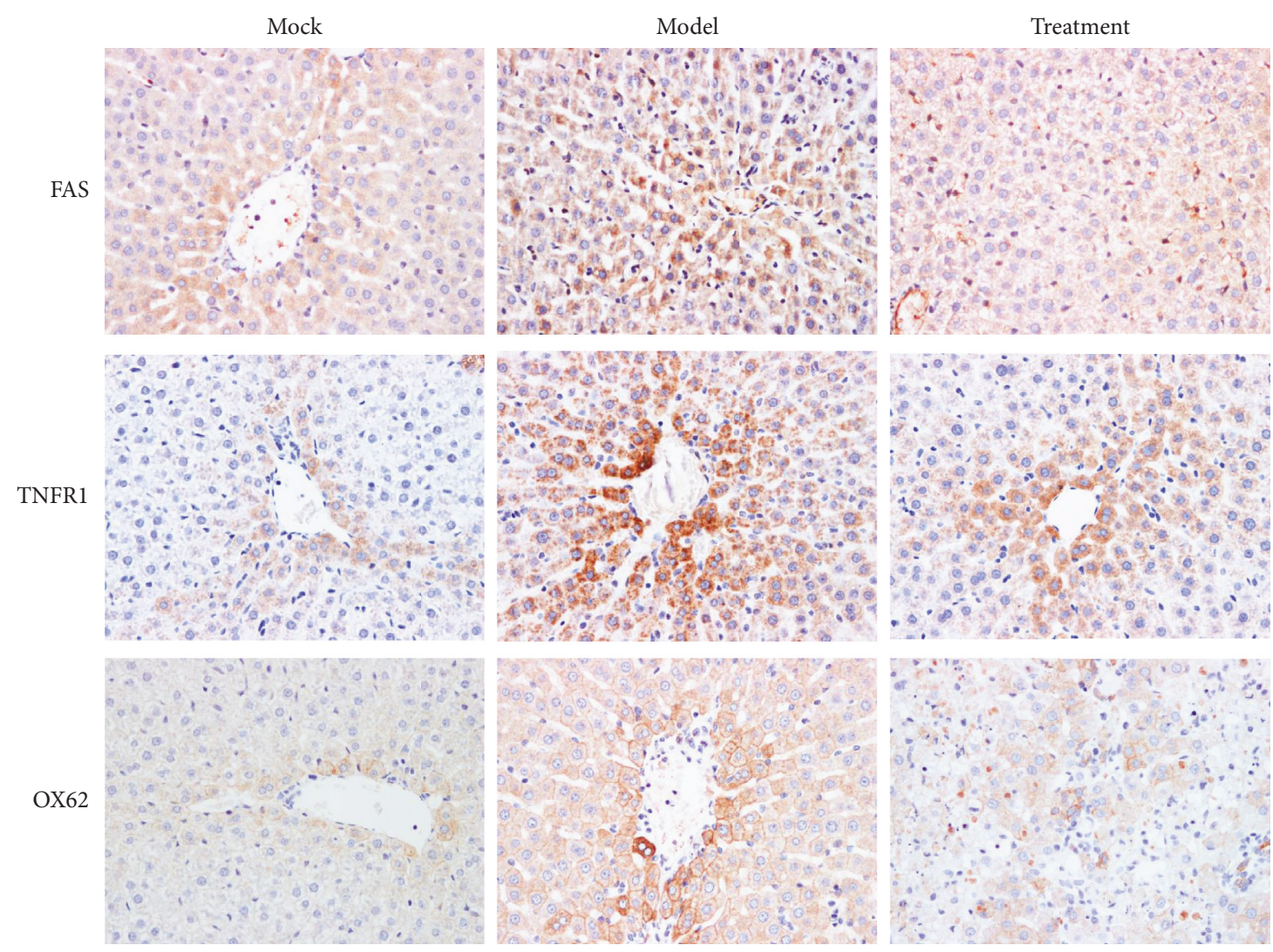

(a)
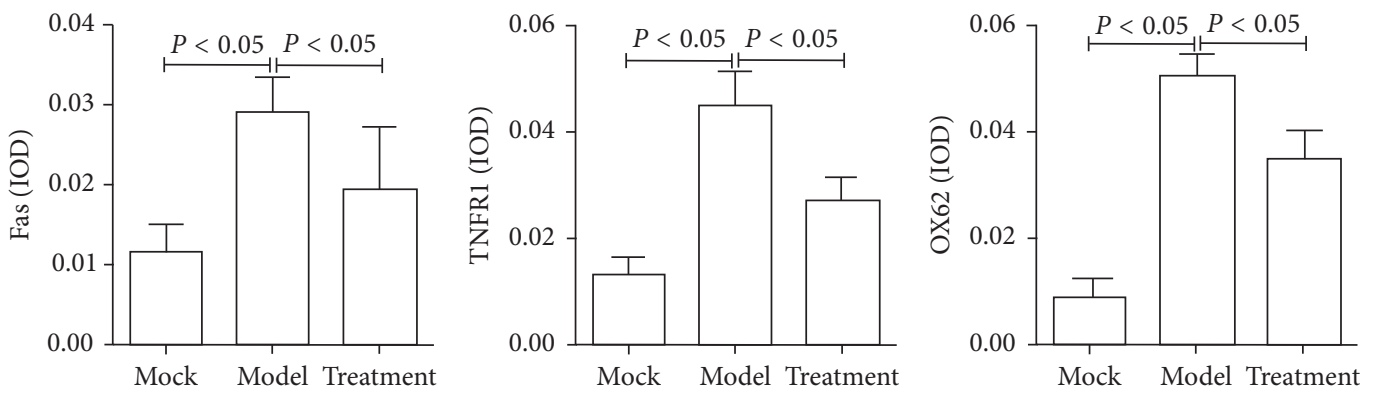

(b)

FIGURE 4: Fas, TNFR1, and OX62 protein expression in rat liver cells detected by immunohistochemistry. (a) Rats were killed, the liver was collected, and Fas, TNFR1, and OX62 were detected by immunohistochemistry (400x magnification). (b) IPP6.0 software was used to analyze the optical density of the staining in the immunohistochemical images. Three 400x magnification images were selected from each section for optical density analysis.

ascribed in Western medicine. At present, more and more studies proved the mechanism of action of the drug to treat liver damage such as antioxidant activity, inflammation reduction, and apoptosis inhibition. It has also been shown to protect the liver in clinical trials, and it is widely used against liver fibrosis [22]. Our research revealed that the Jiedu Huayu granules are especially acting in the immune system.

With the continuous improvement of the basic and clinical research in liver diseases, the understanding of the etiology of diseases, causes, and treatments have also made great progresses, and the clinical treatment on liver diseases has been widely recognized and promoted. For example, the treatment against viral hepatitis changed from a simple treatment of liver-protecting enzymes to the current antiviral treatment and has achieved good clinical results. With the continuous development and promotion of antiviral treatments, some people believe that an antivirus is enough to resolve a viral disease [23]. To solve all the problems of hepatitis B and C, liver protection treatment seems to be a bit redundant [24, 25]. However, the understanding of the pathogenesis of liver diseases revealed that the virus itself does not cause liver damage but is the immune system to be involved. Therefore, the use of antivirus and hepatoprotective agents is more beneficial. 


\section{Data Availability}

The data used to support the findings of this study cannot be shared at this time as the data also form part of an ongoing study.

\section{Conflicts of Interest}

The authors declare that there are no conflicts of interests.

\section{Authors' Contributions}

All authors equally contributed to this paper regarding conception and design of the study, literature review and analysis, drafting, critical revision, editing, and approval of the final version.

\section{Acknowledgments}

This study was financially supported by the National Nonprofit Institute Research Grant for Institute of Basic Theory for Chinese Medicine, CACMS (YZ-1615), Hanmin Li Famous Old Chinese Medicine Experts Inheritance Studio, National Natural Science Foundation of China (nos. 81774236 and 81973669), Guangxi Liver Disease Clinical Research Center of Traditional Chinese Medicine, National Clinical Research Base of Traditional Chinese Medicine (Guangxi Province), Guangxi Natural Science Foundation (2017GXNSFBA198200 and 2018GXNSFAA281047), and Innovation Team Project of Guangxi Natural Science Foundation (2018GXNSFGA281002).

\section{References}

[1] J.-M. Hwang, T.-H. Tseng, Y.-Y. Tsai et al., "Protective effects of baicalein on tert-butyl hydroperoxide-induced hepatic toxicity in rat hepatocytes," Journal of Biomedical Science, vol. 12, no. 2, pp. 389-397, 2005.

[2] R. Kumar, V. Shalimar, V. Bhatia et al., "Antituberculosis therapy-induced acute liver failure: magnitude, profile, prognosis, and predictors of outcome," Hepatology, vol. 51, no. 5, pp. 1665-1674, 2010.

[3] Y. Zou, Y. Yang, J. Li, W. Li, and Q. Wu, "Prevention of hepatic injury by a traditional Chinese formulation, BJ-JN, in mice treated with Bacille-Calmette-Guérin and lipopolysaccharide," Journal of Ethnopharmacology, vol. 107, no. 3, pp. 442-448, 2006.

[4] T. Nakama, S. Hirono, A. Moriuchi et al., "Etoposide prevents apoptosis in mouse liver with ?-galactosamine/lipopolysaccharide-induced fulminant hepatic failure resulting in reduction of lethality," Hepatology, vol. 33, no. 6, pp. 1441-1450, 2001.

[5] M. Arvelo, J. T. Cooper, C. Longo et al., "A20 protects mice from D-galactosamine/lipopolysaccharide acute toxic lethal hepatitis," Hepatology, vol. 35, no. 3, pp. 535-543, 2002.

[6] N. Enomoto, K. Ikejima, S. Yamashina et al., "Kupffer cell sensitization by alcohol involves increased permeability to gut-derived endotoxin," Alcoholism: Clinical and Experimental Research, vol. 25, no. 6 Suppl, pp. 51S-54S, 2001.

[7] M. D. Josephs, F. R. Bahjat, K. Fukuzuka et al., "Lipopolysaccharide and d-galactosamine-induced hepatic injury is mediated by TNF- $\alpha$ and not by Fas ligand," American Journal of Physiology-Regulatory, Integrative and Comparative Physiology, vol. 278, no. 5, pp. R1196-R1201, 2000.

[8] A. Mignon, N. Rouquet, M. Fabre et al., "LPS challenge in D-galactosamine-sensitized mice accounts for caspase-dependent fulminant hepatitis, not for septic shock," American Journal of Respiratory and Critical Care Medicine, vol. 159, no. 4, pp. 1308-1315, 1999.

[9] D. Kägi, B. Ledermann, K. Bürki et al., "Cytotoxicity mediated by $\mathrm{T}$ cells and natural killer cells is greatly impaired in perforin-deficient mice," Nature, vol. 369, no. 6475, pp. 31-37, 1994.

[10] L. Golden-Mason, B. Palmer, J. Klarquist, J. A. Mengshol, N. Castelblanco, and H. R. Rosen, "Upregulation of PD-1 expression on circulating and intrahepatic hepatitis $C$ virusspecific $\mathrm{CD}^{+} \mathrm{T}$ cells associated with reversible immune dysfunction," Journal of Virology, vol. 81, no. 17, pp. 9249-9258, 2007.

[11] D. Xu, J. Fu, L. Jin et al., "Circulating and liver resident $\mathrm{CD} 4{ }^{+} \mathrm{CD} 25^{+}$regulatory $\mathrm{T}$ cells actively influence the antiviral immune response and disease progression in patients with hepatitis B," The Journal of Immunology, vol. 177, no. 1, pp. 739-747, 2006.

[12] .S. Wang, N. Wang, and N. Tang, "Clinical study of leading integrated Chinese and Western medicine treatment of chronic on acute liver failure in the Jieduhuayu granule," Chinese Journal of Integrated Traditional and Western Medicine on Liver Diseases, vol. 24, no. 4, p. 3, 2014.

[13] Y. Chen, D. Mao, J. Yu et al., "Effect of Jieduhuayu granule on the expression of caspase-3mRNA in liver cells of acute liver failure rat," Lishizhen Medicine and Materia Medica Research, vol. 20, no. 9, pp. 2251-2254, 2009.

[14] C. Yao, G. Huang, M. Wang et al., "Chinese herbal medicine formula Jieduhuayu granules improves cognitive and neurophysiological functions in patients with cirrhosis who have minimal hepatic encephalopathy: a randomized controlled trial," Complementary Therapies in Medicine, vol. 22, no. 6, pp. 977-985, 2014.

[15] M. Wang, Q. Shi, R. Zhang, H. Qiu, D. Mao, and F. Long, "Herbal compound "Jiedu Huayu" reduces liver injury in rats via regulation of IL-2, TLR4, and PCNA expression levels," Evidence-Based Complementary and Alternative Medicine, vol. 2017, Article ID 9819350, 9 pages, 2017.

[16] M. P. Jammal, A. A. Da Silva, A. M. Filho et al., "Immunohistochemical staining of tumor necrosis factor- $\alpha$ and interleukin-10 in benign and malignant ovarian neoplasms," Oncology Letters, vol. 9, no. 2, pp. 979-983, 2015.

[17] Y. Liu, M. Wang, D. Mao, and Y. Pei, "Effect of Jiedu Huayu granule on peripheral CTL and treg cells in rats with liver failure," Lishizhen Medicine and Materia Medica Research, vol. 29, no. 6, pp. 1290-1293.

[18] Y. Pei, D. Mao, M. Wang, and R. Zhang, "Effect of Jiedu Huayu granule on the expression of OX62, Fas and TNFR1 in rats with acute liver failure," Journal of Guangzhou University of Traditional Chinese Medicine, vol. 35, no. 3, pp. 471-476.

[19] C. Bernsmeier, A. Singanayagam, V. C. Patel, J. Wendon, and C. G. Antoniades, "Immunotherapy in the treatment and prevention of infection in acute-on-chronic liver failure," Immunotherapy, vol. 7, no. 6, pp. 641-654, 2015.

[20] J. Clària, V. Arroyo, and R. Moreau, "The acute-on-chronic liver failure syndrome, or when the innate immune system goes astray," The Journal of Immunology, vol. 197, no. 10, pp. 3755-3761, 2016.

[21] P. J. Trivedi and D. H. Adams, "Gut-liver immunity," Journal of Hepatology, vol. 64, no. 5, pp. 1187-1189, 2015. 
[22] Y. He, L. Jin, J. Wang, Z. Yan, T. Chen, and Y. Zhao, "Mechanisms of fibrosis in acute liver failure," Liver International, vol. 35, no. 7, pp. 1877-1885, 2015.

[23] Z. Cao, F. Li, X. Xiang et al., "Circulating cell death biomarker: good candidates of prognostic indicator for patients with hepatitis B virus related acute-on-chronic liver failure," Scientific Reports, vol. 5, no. 1, Article ID 14240, 2015.

[24] M.-F. Yuen, "Anti-viral therapy in hepatitis B virus reactivation with acute-on-chronic liver failure," Hepatology International, vol. 9, no. 3, pp. 373-377, 2015.

[25] M. Masetti, D. Magalotti, E. Martino, P. Andreone, A. Scuteri, and M. Zoli, "A case of acute liver failure during ritonavirboosted paritaprevir, ombitasvir and dasabuvir therapy in a patient with HCV genotype 1b cirrhosis," Journal of Gastrointestinal and Liver Diseases, vol. 25, no. 4, pp. 559-561, 2016. 


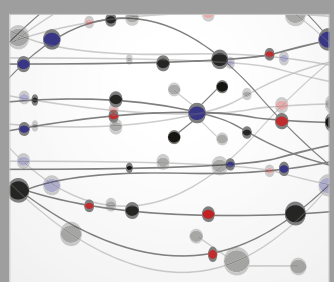

The Scientific World Journal
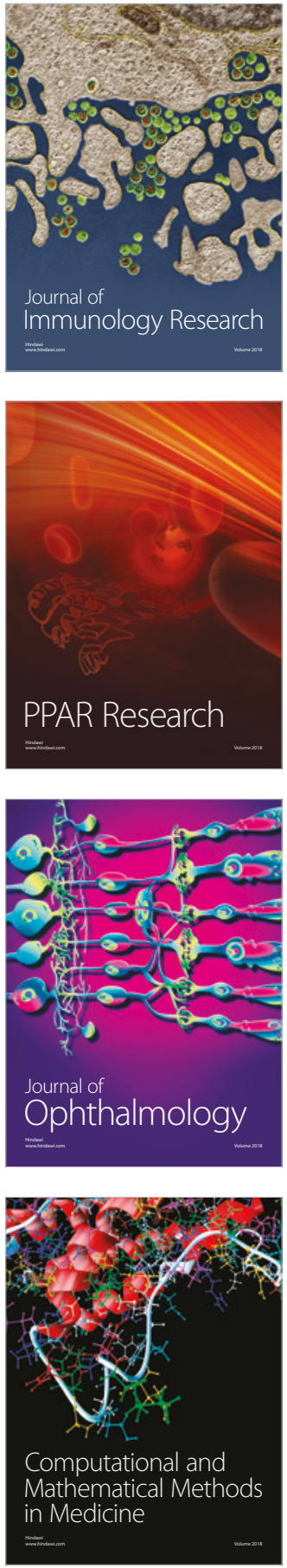

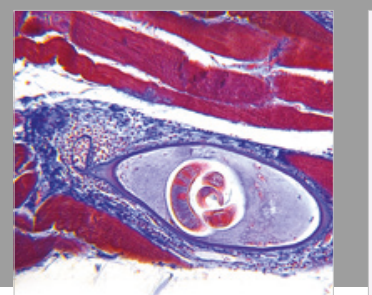

Gastroenterology Research and Practice

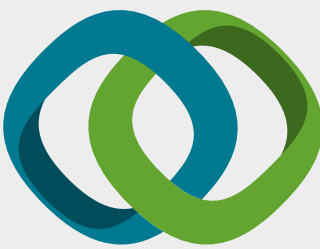

\section{Hindawi}

Submit your manuscripts at

www.hindawi.com
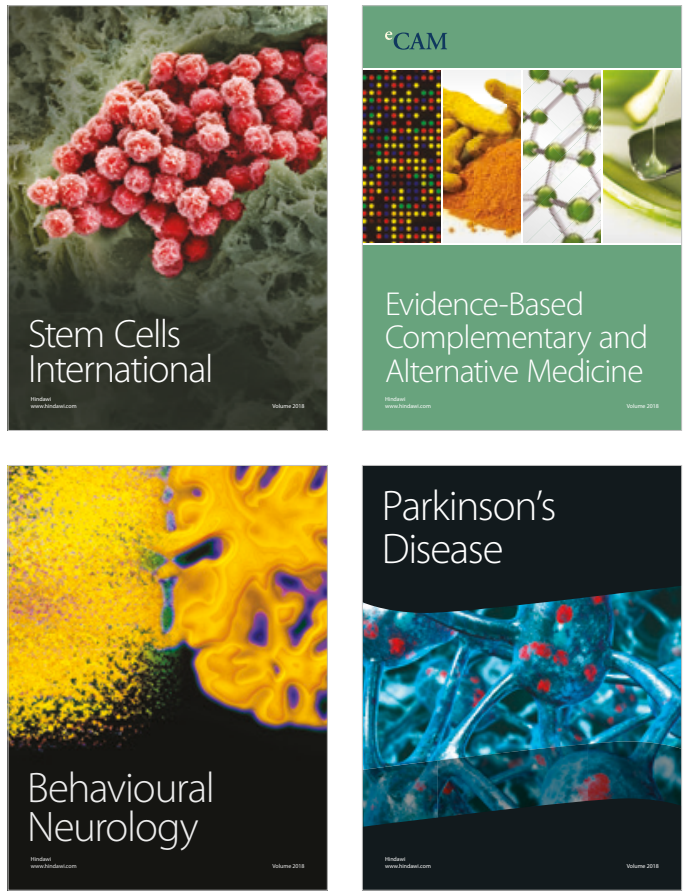

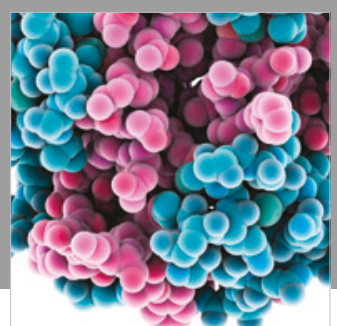

ournal of

Diabetes Research

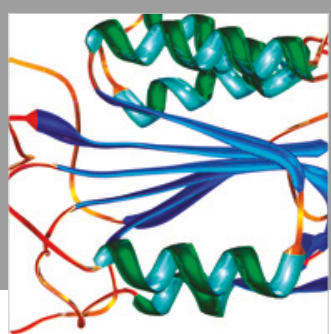

Disease Markers
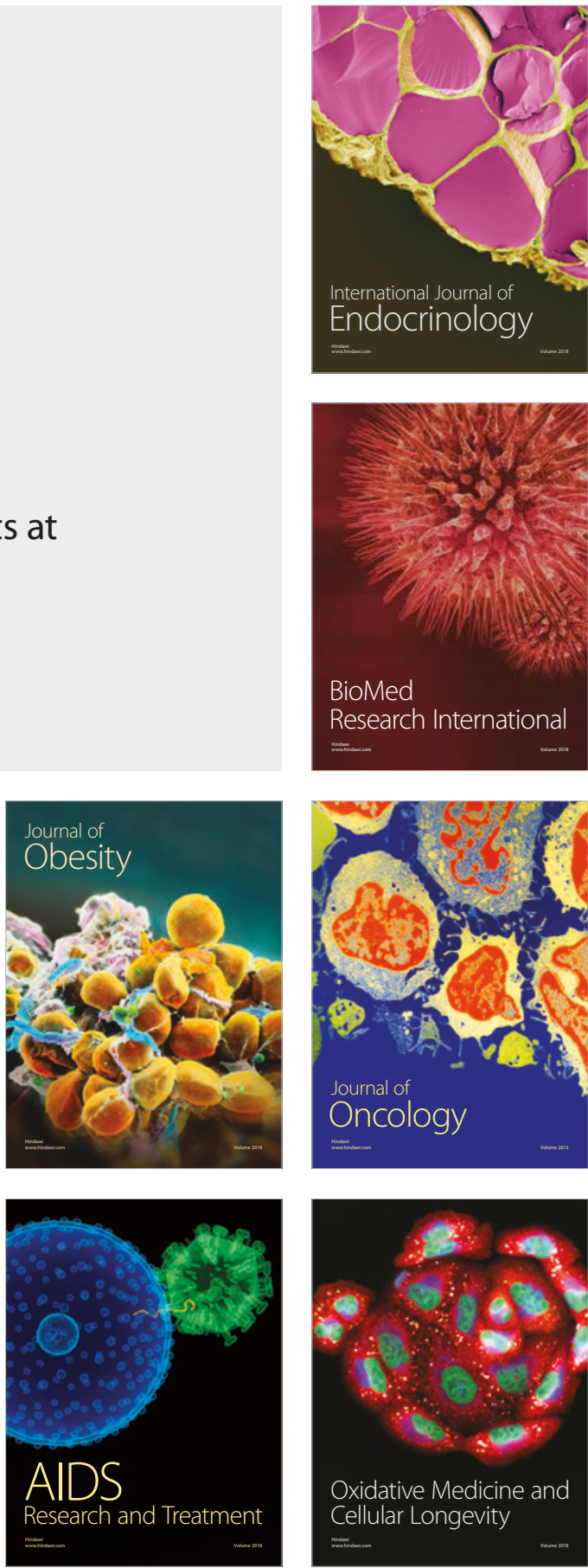